\title{
On the Iterative Method for the System of Nonlinear Matrix Equations
}

\author{
Asmaa M. Al-Dubiban \\ Faculty of Science and Arts, Qassim University, Buraydah 51431, P.O. Box 1162, Saudi Arabia \\ Correspondence should be addressed to Asmaa M. Al-Dubiban; dr.dubiban@hotmail.com
}

Received 15 November 2012; Accepted 20 February 2013

Academic Editor: Mohammad T. Darvishi

Copyright (C) 2013 Asmaa M. Al-Dubiban. This is an open access article distributed under the Creative Commons Attribution License, which permits unrestricted use, distribution, and reproduction in any medium, provided the original work is properly cited.

The positive definite solutions for the system of nonlinear matrix equations $X+A^{*} Y^{-n} A=I, Y+B^{*} X^{-m} B=I$ are considered, where $n, m$ are two positive integers and $A, B$ are nonsingular complex matrices. Some sufficient conditions for the existence of positive definite solutions for the system are derived. Under some conditions, an iterative algorithm for computing the positive definite solutions for the system is proposed. Also, the estimation of the error is obtained. Finally, some numerical examples are given to show the efficiency of the proposed iterative algorithm.

\section{Introduction}

Linear and nonlinear matrix equations have been widely used for solving many problems in several areas such as control theory, optimal control, optimization control, stability theory, communication system, dynamic programming, signal processing, and stochastic filtering and statistics, [13]. Many authors studied the existence of solutions for several classes of the matrix equations (see, e.g., [4-14]), in particular, Lyapunov matrix equation [15], Sylvester matrix equations [11, 14], algebraic Riccati equations [3], some special case of linear and nonlinear matrix equations [16-21], and coupled matrix equations [22-24].

In recent years, many types of algebraic Riccati equations have been the subject of great activity, the aim being to achieve a fast and reliable algorithm that generates numerical positive definite solutions.

In this paper, we will consider the system (Sys.) of nonlinear matrix equations that can be expressed in the form:

$$
\begin{aligned}
& X+A^{*} Y^{-n} A=I, \\
& Y+B^{*} X^{-m} B=I,
\end{aligned}
$$

where $n, m$ are two positive integers, $X, Y$ are $r \times r$ unknown matrices, $I$ is the $r \times r$ identity matrix, and $A, B$ are nonsingular matrices. All matrices are defined over the complex field.
The system of nonlinear matrix equations with the form of (1) is a special case of the system of algebraic discrete-type Riccati equations of the form:

$$
\begin{aligned}
X_{i}= & V_{i}^{*} X_{i} V_{i}+Q_{i} \\
& -\left(V_{i}^{*} X_{i} B_{i}+A_{i}\right)\left(R_{i}+B_{i}^{*} X_{i} B_{i}\right)^{-1}\left(B_{i}^{*} X_{i} V_{i}+A_{i}^{*}\right),
\end{aligned}
$$

where $i=1,2, \ldots, k,[2,3]$. The efficient numerical solutions for some special case of the system (2) have been extensively studied by several authors [4-10, 22-26]. For example, Mukaidani [22] proposed a new algorithm for solving crosscoupled sign-indefinite algebraic Riccati equations for weakly coupled large-scale systems, while in $[4,5]$ Al-Dubiban has studied special cases of Sys. (2) by obtained sufficient conditions for the existence of positive definite solutions for the systems and proposed iterative algorithms to calculate the solutions. In [10], Davies proposed upper bounds for the sum of the maximal eigenvalues of the solutions of the continuous and discrete coupled algebraic Riccati equations. In [25], Ivanov has studied a set of discrete-time coupled algebraic Riccati equations which arise in quadratic optimal control and proposed two iterations for computing a symmetric solution of this system.

In this paper, we derive the sufficient conditions of the existence of solutions for the Sys. (1). We introduce an 
iterative algorithm to obtain the positive definite solutions of Sys. (1). We discuss the convergence of this iterative algorithm. Finally, some numerical examples are given to illustrate the efficiency for suggested algorithm.

The following notations are used throughout the rest of the paper. The notation $A \geq 0(A>0)$ means that $A$ is positive semidefinite (positive definite), $A^{\star}$ denotes the complex conjugate transpose of $A$, and $I$ is the identity matrix. Moreover, $A \geq B(A>B)$ is used as a different notation for $A-B \geq 0(A-B>0)$. We denote by $\rho(A)$ the spectral radius of $A ; \lambda_{r}(X), \mu_{r}(Y)$ represent the eigenvalues of $X$ and $Y$, respectively. The norm used in this paper is the spectral norm of the matrix $A$; that is, $\|A\|=\sqrt{\rho\left(A A^{\star}\right)}$ unless otherwise noted.

\section{Main Theorems}

In this section, we will introduce an iterative algorithm which is applicable for computing the positive definite solutions of the Sys. (1). We start with some results which will be used throughout this paper.

Lemma 1 (see [27, 28]). If $P>Q>0$ (or $P \geq Q>0$ ), then $P^{\alpha}>Q^{\alpha}$ (or $P^{\alpha} \geq Q^{\alpha}>0$ ) for all $\alpha \in(0,1]$, and $P^{\alpha}<Q^{\alpha}$ (or $\left.0<P^{\alpha} \leq Q^{\alpha}\right)$ for all $\alpha \in[-1,0)$.

Theorem 2 (see [29]). Let the matrices $P, Q$, and $R$ be positive definite $r \times r$ matrices, such that the integral

$$
\int_{0}^{\infty} e^{P t} R e^{\mathrm{Q} t} d t
$$

exists and

$$
\lim _{t \rightarrow \infty} e^{P t} R e^{\mathrm{Q} t}=0
$$

then the matrix

$$
Z=-\int_{0}^{\infty} e^{P t} R e^{Q t} d t
$$

is the solution of the matrix equation:

$$
P Z+Z Q=R
$$

The solution of Sys. (1) can be found by the following iterative algorithm.

Algorithm 3.

$$
\begin{gathered}
X_{0}=Y_{0}=\beta I, \quad X_{s+1}=\left[B\left(I-Y_{s}\right)^{-1} B^{*}\right]^{1 / m}, \\
Y_{s+1}=\left[A\left(I-X_{s}\right)^{-1} A^{*}\right]^{1 / n}, \quad s=0,1,2, \ldots
\end{gathered}
$$

Theorem 4. If there exist numbers $\alpha$, $\beta$ satisfying $0<\alpha<\beta \leq$ $\min \{n /(n+1), m /(m+1)\}$, and the following conditions hold:

(i) $\alpha^{n}(1-\alpha) I<A A^{*}<\beta^{n}(1-\beta) I$,

(ii) $\alpha^{m}(1-\alpha) I<B B^{*}<\beta^{m}(1-\beta) I$, then the sequences $\left\{X_{s}\right\},\left\{Y_{s}\right\}$ defined by Algorithm 3 converge to a positive definite solution $(X, Y)$ of Sys. (1).

Proof. From Algorithm 3, we get

$$
\begin{aligned}
X_{1} & =\left[B(I-\beta I)^{-1} B^{*}\right]^{1 / m} \\
& =\left[\frac{1}{(1-\beta)} B B^{*}\right]^{1 / m}<\left[\frac{\beta^{m}(1-\beta)}{(1-\beta)} I\right]^{1 / m}=\beta I=X_{0} .
\end{aligned}
$$

Also, we have

$$
\begin{aligned}
X_{1} & =\left[B(I-\beta I)^{-1} B^{*}\right]^{1 / m}=\left[\frac{1}{(1-\beta)} B B^{*}\right]^{1 / m} \\
& >\left[\frac{1}{(1-\alpha)} B B^{*}\right]^{1 / m}>\left[\frac{\alpha^{m}(1-\alpha)}{(1-\alpha)} I\right]^{1 / m}=\alpha I .
\end{aligned}
$$

That is, $X_{0}>X_{1}>\alpha I$, similarly we get

$$
\begin{aligned}
Y_{1} & =\left[A(I-\beta I)^{-1} A^{*}\right]^{1 / n} \\
& =\left[\frac{1}{(1-\beta)} A A^{*}\right]^{1 / n}<\left[\frac{\beta^{n}(1-\beta)}{(1-\beta)} I\right]^{1 / n}=\beta I=Y_{0} .
\end{aligned}
$$

Also, we have

$$
\begin{aligned}
Y_{1} & =\left[A(I-\beta I)^{-1} A^{*}\right]^{1 / n} \\
& =\left[\frac{1}{(1-\beta)} A A^{*}\right]^{1 / n}>\left[\frac{1}{(1-\alpha)} A A^{*}\right]^{1 / n} \\
& >\left[\frac{\alpha^{n}(1-\alpha)}{(1-\alpha)} I\right]^{1 / n}=\alpha I .
\end{aligned}
$$

That is, $Y_{0}>Y_{1}>\alpha I$.

Suppose that

$$
X_{s-1}>X_{s}>\alpha I, \quad Y_{s-1}>Y_{s}>\alpha I .
$$

Now, we will prove that $X_{s}>X_{s+1}>\alpha I$ and $Y_{s}>Y_{s+1}>\alpha I$. By using the inequalities (12), we have

$$
X_{s+1}=\left[B\left(I-Y_{s}\right)^{-1} B^{*}\right]^{1 / m}<\left[B\left(I-Y_{s-1}\right)^{-1} B^{*}\right]^{1 / m}=X_{s} .
$$

Also, we have

$$
\begin{aligned}
X_{s+1} & =\left[B\left(I-Y_{s}\right)^{-1} B^{*}\right]^{1 / m}>\left[B(I-\alpha I)^{-1} B^{*}\right]^{1 / m} \\
& =\left[\frac{1}{(1-\alpha)} B B^{*}\right]^{1 / m}>\left[\frac{\alpha^{m}(1-\alpha)}{(1-\alpha)} I\right]^{1 / m}=\alpha I .
\end{aligned}
$$

Similarly, we get

$$
Y_{s+1}=\left[A\left(I-X_{s}\right)^{-1} A^{*}\right]^{1 / n}<\left[A\left(I-X_{s-1}\right)^{-1} A^{*}\right]^{1 / n}=Y_{s} .
$$


Also, we have

$$
\begin{aligned}
Y_{s+1} & =\left[A\left(I-X_{s}\right)^{-1} A^{*}\right]^{1 / n}>\left[A(I-\alpha I)^{-1} A^{*}\right]^{1 / n} \\
& =\left[\frac{1}{(1-\alpha)} A A^{*}\right]^{1 / n}>\left[\frac{\alpha^{n}(1-\alpha)}{(1-\alpha)} I\right]^{1 / n}=\alpha I .
\end{aligned}
$$

Therefore, the inequalities (12) are true for all $s=1,2,3, \ldots$.

Hence, the sequences $\left\{X_{s}\right\},\left\{Y_{s}\right\}$ are monotonically decreasing and bounded from below by the matrix $\alpha I$. Consequently, the sequences converge to a positive definite $\operatorname{limit}(X, Y)$ which is a solution of Sys. (1).

Theorem 5. If there exist numbers $\alpha, \beta$ satisfying $0<\alpha<\beta \leq$ $\min \{n /(n+1), m /(m+1)\}$, and the following conditions hold:

(i) $\alpha^{n}(1-\alpha) I<A A^{*}<\beta^{n}(1-\beta) I$,

(ii) $\alpha^{m}(1-\alpha) I<B B^{*}<\beta^{m}(1-\beta) I$,

(iii) $q=\beta^{\left(2^{k}+2^{t}\right)} /(2 \alpha)^{(k+t)}(1-\beta)^{2}<1$,

where $m=2^{k}, n=2^{t}$, then Sys. (1) has a positive definite solution $(X, Y)$ which satisfies

$$
\begin{gathered}
\left\|X_{s}-X\right\| \leq q\left\|X_{s-2}-X\right\| \leq \cdots \leq(q)^{s / 2}(\beta-\alpha), \\
\left\|Y_{s}-Y\right\| \leq q\left\|Y_{s-2}-Y\right\| \leq \cdots \leq(q)^{s / 2}(\beta-\alpha) .
\end{gathered}
$$

Proof. From Theorem 4, the two sequences $\left\{X_{s}\right\},\left\{Y_{s}\right\}$ defined by Algorithm 3 are convergent to a positive definite solution $(X, Y)$ of Sys. (1). We compute the spectral norm of the matrices $X_{s}-X, Y_{s}-Y$. For that, we have

$$
\left\|X_{s}-X\right\|=\left\|\sqrt[m]{B\left(I-Y_{s-1}\right)^{-1} B^{*}}-\sqrt[m]{B(I-Y)^{-1} B^{*}}\right\| .
$$

We denote

$$
P_{1}=B\left(I-Y_{s-1}\right)^{-1} B^{*}, \quad Q_{1}=B(I-Y)^{-1} B^{*} .
$$

We use the following equality:

$$
\begin{aligned}
& \sqrt[m]{P_{1}}\left(\sqrt[m]{P_{1}}-\sqrt[m]{Q_{1}}\right)+\left(\sqrt[m]{P_{1}}-\sqrt[m]{Q_{1}}\right) \sqrt[m]{Q_{1}} \\
& =\sqrt[m]{P_{1}}-\sqrt[m]{Q_{1}} .
\end{aligned}
$$

Since $Y_{s}>Y>0$ for each $s=0,1,2, \ldots$, then by using Lemma 1 we have the matrix $D_{1}=\sqrt[m]{P_{1}}-\sqrt[m]{Q_{1}}$ being a positive definite solution of the matrix equation:

$$
\sqrt[m]{P_{1}} D_{1}+D_{1} \sqrt[m]{Q_{1}}=\sqrt[m / 2]{P_{1}}-\sqrt[m]{Q_{1}} .
$$

According to Theorem 2, we have

$$
D_{1}=\int_{0}^{\infty} e^{-\sqrt[m]{P_{1}} t}\left(\sqrt[m / 2]{P_{1}}-\sqrt[m / 2]{Q_{1}}\right) e^{-\sqrt[m]{Q_{1}} t} d t
$$

Since $\sqrt[m]{P_{1}}, \sqrt[m]{Q_{1}}$ are positive definite matrices, then the integral (22) exists, and

$$
e^{-\sqrt[m]{P_{1}} t}\left(\sqrt[m / 2]{P_{1}}-\sqrt[m / 2]{Q_{1}}\right) e^{-\sqrt[m]{Q_{1}} t} \longrightarrow 0, \quad \text { as } t \longrightarrow \infty .
$$

By using (18) and (22), we have

$$
\left\|X_{s}-X\right\| \leq \int_{0}^{\infty}\left\|\left(\sqrt[m / 2]{P_{1}}-\sqrt[m / 2]{Q_{1}}\right)\right\|\left\|e^{-\sqrt[m]{P_{1}} t}\right\|\left\|e^{-\sqrt[m]{Q_{1}} t}\right\| d t .
$$

However, $Y_{s}>Y \geq \alpha I$; hence,

$$
\sqrt[m]{P_{1}}>\alpha I, \quad \sqrt[m]{Q_{1}} \geq \alpha I
$$

Then, we have

$$
\begin{aligned}
\left\|X_{s}-X\right\| & \leq\left\|\sqrt[m / 2]{P_{1}}-\sqrt[m / 2]{Q_{1}}\right\| \int_{0}^{\infty}\left\|e^{-\sqrt[m]{P_{1}} t}\right\|\left\|e^{-\sqrt[m]{Q_{1}} t}\right\| d t \\
& \leq\left\|\sqrt[m / 2]{P_{1}}-\sqrt[m / 2]{Q_{1}}\right\| \int_{0}^{\infty}\left\|e^{-\alpha I t}\right\|^{2} d t \\
& =\left\|\sqrt[m / 2]{P_{1}}-\sqrt[m / 2]{Q_{1}}\right\| \int_{0}^{\infty} e^{-2 \alpha t} d t \\
& =\frac{1}{2 \alpha}\left\|\sqrt[m / 2]{P_{1}}-\sqrt[m / 2]{Q_{1}}\right\| \\
& \leq\left(\frac{1}{2 \alpha}\right)^{2}\left\|\sqrt[m / 2]{P_{1}}-\sqrt[m / 2]{Q_{1}}\right\| .
\end{aligned}
$$

After $k$ times as above, we get

$$
\left\|X_{s}-X\right\| \leq\left(\frac{1}{2 \alpha}\right)^{k}\left\|\sqrt[m / 2^{k}]{P_{1}}-\sqrt[m / 2^{k}]{Q_{1}}\right\| .
$$

Let $m=2^{k}$ be special case, then we have

$$
\begin{aligned}
\left\|X_{s}-X\right\| & \leq\left(\frac{1}{2 \alpha}\right)^{k}\left\|P_{1}-Q_{1}\right\| \\
& =\left(\frac{1}{2 \alpha}\right)^{k}\left\|B\left(I-Y_{s-1}\right)^{-1} B^{*}-B(I-Y)^{-1} B^{*}\right\| \\
& =\left(\frac{1}{2 \alpha}\right)^{k}\left\|B\left(I-Y_{s-1}\right)^{-1}\left(Y_{s-1}-Y\right)(I-Y)^{-1} B^{*}\right\| \\
& \leq\left(\frac{1}{2 \alpha}\right)^{k}\|B\|^{2}\left\|\left(I-Y_{s-1}\right)^{-1}\right\|\left\|(I-Y)^{-1}\right\|\left\|Y_{s-1}-Y\right\| .
\end{aligned}
$$

Since $Y<Y_{s-1} \leq \beta I$, then $(I-Y)^{-1}<\left(I-Y_{s-1}\right)^{-1} \leq(1 /(1-$ $\beta)) I$; that is,

$$
\left\|(I-Y)^{-1}\right\|<\left\|\left(I-Y_{s-1}\right)^{-1}\right\| \leq \frac{1}{(1-\beta)} .
$$

Therefore, we get

$$
\left\|X_{s}-X\right\| \leq\left(\frac{1}{2 \alpha}\right)^{k} \frac{\beta^{2^{k}}}{(1-\beta)}\left\|Y_{s-1}-Y\right\| .
$$

Also, we have

$$
\left\|Y_{s}-Y\right\|=\left\|\sqrt[n]{A\left(I-X_{s-1}\right)^{-1} A^{*}}-\sqrt[n]{A(\mathrm{I}-X)^{-1} A^{*}}\right\| .
$$


We denote

$$
P_{2}=A\left(I-X_{s-1}\right)^{-1} A^{*}, \quad Q_{2}=A(I-X)^{-1} A^{*} .
$$

We use the following equality:

$$
\begin{aligned}
& \sqrt[n]{P_{2}}\left(\sqrt[n]{P_{2}}-\sqrt[n]{Q_{2}}\right)+\left(\sqrt[n]{P_{2}}-\sqrt[n]{Q_{2}}\right) \sqrt[n]{Q_{2}} \\
& =\sqrt[n]{\mathrm{P}_{2}}-\sqrt[n]{\mathrm{Q}_{2}} .
\end{aligned}
$$

Since $X_{s}>X>0$ for each $s=0,1,2, \ldots$, then by using Lemma 1 we have the matrix $D_{2}=\sqrt[n]{P_{2}}-\sqrt[n]{Q_{2}}$ being a positive definite solution of the matrix equation:

$$
\sqrt[n]{P_{2}} D_{2}+D_{2} \sqrt[n]{Q_{2}}=\sqrt[n]{P_{2}}-\sqrt[n]{Q_{2}}
$$

According to Theorem 2, we have

$$
D_{2}=\int_{0}^{\infty} e^{-\sqrt[n]{P_{2}} t}\left(\sqrt[n n 2]{P_{2}}-\sqrt[n n 2]{Q_{2}}\right) e^{-\sqrt[n]{Q_{2}} t} d t
$$

Since $\sqrt[n]{P_{2}}, \sqrt[n]{Q_{2}}$ are positive definite matrices, then the integral (35) exists, and

$$
e^{-\sqrt[n]{P_{2}} t}\left(\sqrt[n / 2]{P_{2}}-\sqrt[n]{Q_{2}}\right) e^{-\sqrt[n]{Q_{2}} t} \longrightarrow 0, \quad \text { as } t \longrightarrow \infty .
$$

By using (31) and (35), we have

$$
\left\|Y_{s}-Y\right\| \leq \int_{0}^{\infty}\left\|\left(\sqrt[n / 2]{P_{2}}-\sqrt[n]{Q_{2}}\right)\right\|\left\|e^{-\sqrt[n]{P_{2}} t}\right\|\left\|e^{-\sqrt[n]{Q_{2}} t}\right\| d t
$$

However, $X_{s}>X \geq \alpha I$; hence,

$$
\sqrt[n]{P_{2}}>\alpha I, \quad \sqrt[n]{Q_{2}} \geq \alpha I
$$

Then, we have

$$
\begin{aligned}
\left\|Y_{s}-Y\right\| & \leq\left\|\sqrt[n / 2]{P_{2}}-\sqrt[n / 2]{Q_{2}}\right\| \int_{0}^{\infty}\left\|e^{-\sqrt[n]{P_{2}} t}\right\|\left\|e^{-\sqrt[n]{Q_{2}} t}\right\| d t \\
& \leq\left\|\sqrt[n / 2]{P_{2}}-\sqrt[n / 2]{Q_{2}}\right\| \int_{0}^{\infty}\left\|e^{-\alpha I t}\right\|^{2} d t \\
& =\left\|\sqrt[n / 2]{P_{2}}-\sqrt[n / 2]{Q_{2}}\right\| \int_{0}^{\infty} e^{-2 \alpha t} d t \\
& =\frac{1}{2 \alpha}\left\|\sqrt[n / 2]{P_{2}}-\sqrt[n / 2]{Q_{2}}\right\| \\
& \leq\left(\frac{1}{2 \alpha}\right)^{2}\left\|\sqrt[n / 2]{P_{2}}-\sqrt[n / 2]{Q_{2}}\right\| .
\end{aligned}
$$

After $t$ times as above, we get

$$
\left\|Y_{s}-Y\right\| \leq\left(\frac{1}{2 \alpha}\right)^{t}\left\|\sqrt[n / 2]{P_{2}}-\sqrt[n / 2 t]{Q_{2}}\right\|
$$

Let $n=2^{t}$ be special case, then we have

$$
\begin{aligned}
\left\|Y_{s}-Y\right\| & \leq\left(\frac{1}{2 \alpha}\right)^{t}\left\|P_{2}-Q_{2}\right\| \\
& =\left(\frac{1}{2 \alpha}\right)^{t}\left\|A\left(I-X_{s-1}\right)^{-1} A^{*}-A(I-X)^{-1} A^{*}\right\| \\
& =\left(\frac{1}{2 \alpha}\right)^{t}\left\|A\left(I-X_{s-1}\right)^{-1}\left(X_{s-1}-X\right)(I-X)^{-1} A^{*}\right\| \\
& \leq\left(\frac{1}{2 \alpha}\right)^{t}\|A\|^{2}\left\|\left(I-X_{s-1}\right)^{-1}\right\|\left\|(I-X)^{-1}\right\|\left\|X_{s-1}-X\right\| .
\end{aligned}
$$

Since $X<X_{s-1} \leq \beta I$, then $(I-X)^{-1}<\left(I-X_{s-1}\right)^{-1} \leq$ $(1 /(1-\beta)) I$; that is,

$$
\left\|(I-X)^{-1}\right\|<\left\|\left(I-X_{s-1}\right)^{-1}\right\| \leq \frac{1}{(1-\beta)} .
$$

Therefore, we get

$$
\left\|Y_{s}-Y\right\| \leq\left(\frac{1}{2 \alpha}\right)^{t} \frac{\beta^{2^{t}}}{(1-\beta)}\left\|X_{s-1}-X\right\| .
$$

By using (43) in (30) and (30) in (43), we have

$$
\begin{gathered}
\left\|X_{s}-X\right\| \leq q\left\|X_{s-2}-X\right\| \leq \cdots \leq(q)^{s / 2}(\beta-\alpha), \\
\left\|Y_{s}-Y\right\| \leq q\left\|Y_{s-2}-Y\right\| \leq \cdots \leq(q)^{s / 2}(\beta-\alpha),
\end{gathered}
$$

which completes the proof.

\section{Numerical Examples}

We will give some numerical examples for computing the positive definite solution of the Sys. (1). The solution is computed for some different matrices $A, B$ with different orders. Denote by $X, Y$ the solutions which are obtained by Algorithm 3 and $\epsilon_{1}(X)=\left\|X-X_{s}\right\|, \epsilon_{1}(Y)=\left\|Y-Y_{s}\right\|$.

For computing $Z^{1 / n}$ for all $1 / n \in(0,1]$, we use the iterative algorithm.

Algorithm 6.

$$
\begin{array}{r}
W_{0} Z=Z W_{0}, \quad W_{s+1}=\frac{1}{n}\left[(n-1) W_{s}+W_{s}^{1-n} Z\right], \\
s=0,1,2, \ldots .
\end{array}
$$

See [30].

Example 7. Consider Sys. (1) with $n=3, m=2, \beta=0.5$, and normal matrices

$$
\begin{aligned}
& A=\operatorname{diag}[0.2,0.2,0.5], \\
& B=\operatorname{diag}[0.4,0.3,0.1] .
\end{aligned}
$$


TABLE 1: Error analysis for Example 7.

\begin{tabular}{lcc}
\hline$s$ & $\epsilon_{1}(X)$ & $\epsilon_{1}(Y)$ \\
\hline 0 & $3.25515 E-01$ & $1.71540 E-01$ \\
2 & $1.90010 E-01$ & $1.28914 E-01$ \\
4 & $4.65789 E-02$ & $3.11069 E-02$ \\
6 & $8.05431 E-03$ & $5.57630 E-03$ \\
8 & $1.25249 E-03$ & $8.71099 E-04$ \\
10 & $1.90796 E-04$ & $1.32785 E-04$ \\
12 & $2.89695 E-05$ & $2.01634 E-05$ \\
14 & $4.39639 E-06$ & $3.06002 E-06$ \\
16 & $6.67142 E-07$ & $4.64353 E-07$ \\
18 & $1.01236 E-07$ & $7.04637 E-08$ \\
20 & $1.53621 E-08$ & $1.06926 E-08$ \\
\hline
\end{tabular}

By using Algorithms 3 and 6, we have

$$
\begin{gathered}
X=\operatorname{diag}[0.535247,0.388225,0.174485], \\
Y=\operatorname{diag}[0.441516,0.402862,0.67154]
\end{gathered}
$$

The results are given in Table 1.

Example 8. Consider Sys. (1) with $n=5, m=4, \beta=0.4$ and normal matrices

$$
\begin{gathered}
A=\operatorname{diag}\left[\frac{-7}{2 r^{2}}, \frac{-6}{2 r^{2}}, \ldots, \frac{r-8}{2 r^{2}}\right], \\
B=\operatorname{diag}\left[\frac{1}{8+r}, \frac{2}{8+r}, \ldots, \frac{r}{8+r}\right] .
\end{gathered}
$$

By using Algorithms 3 and 6, we have;

$$
\text { When } r=4 \text {, }
$$

$X=\operatorname{diag}[0.362018,0.510342,0.620206,0.707524]$,

$Y=\operatorname{diag}[0.595686,0.590503,0.577588,0.556604]$.

When $r=7$,

$$
\begin{array}{r}
X=\operatorname{diag}[0.290121,0.408797,0.497892, \\
0.570656,0.631828,0.68313,0.723835]
\end{array}
$$$$
Y=\operatorname{diag}[0.372659,0.363432,0.349091 \text {, }
$$$$
0.329437,0.302795,0.265305,0.206669] \text {. }
$$

The results are given in Table 2 .

Example 9. Consider Sys. (1) with $n=2, m=2, \beta=0.4$, and matrices

$$
A=\left(\begin{array}{cc}
0.2 & -0.1 \\
0.1 & 0.2
\end{array}\right), \quad B=\left(\begin{array}{cc}
-0.4 & 0.1 \\
0.2 & 0.3
\end{array}\right)
$$

TABLE 2: Error analysis for Example 8.

\begin{tabular}{cccc}
\hline$r$ & $s$ & $\epsilon_{1}(X)$ & $\epsilon_{1}(Y)$ \\
\hline & 0 & $3.07524 E-01$ & $1.95686 E-01$ \\
& 2 & $2.24755 E-01$ & $1.44449 E-01$ \\
& 4 & $5.31418 E-02$ & $5.07784 E-02$ \\
& 6 & $1.08011 E-02$ & $1.03261 E-02$ \\
4 & 8 & $1.75364 E-03$ & $1.66592 E-03$ \\
& 10 & $2.69238 E-04$ & $2.55471 E-04$ \\
& 12 & $4.09499 E-05$ & $3.88490 E-05$ \\
& 14 & $6.21930 E-06$ & $5.90006 E-06$ \\
& 16 & $9.44353 E-07$ & $8.95875 E-07$ \\
& 18 & $1.43388 E-07$ & $1.36027 E-07$ \\
& 20 & $2.17715 E-08$ & $2.06538 E-08$ \\
\hline & 0 & $3.23835 E-01$ & $1.93331 E-01$ \\
& 2 & $3.64875 E-01$ & $4.33678 E-01$ \\
& 4 & $1.13231 E-01$ & $2.06657 E-01$ \\
7 & 6 & $3.42800 E-02$ & $6.90864 E-02$ \\
& 8 & $6.95159 E-03$ & $7.88070 E-03$ \\
& 10 & $3.42099 E-04$ & $2.93641 E-04$ \\
12 & $1.18241 E-05$ & $1.00690 E-05$ \\
14 & $4.03846 E-07$ & $3.43808 E-07$ \\
16 & $1.37875 E-08$ & $1.17377 E-08$ \\
\hline
\end{tabular}

TABLE 3: Error analysis for Example 9.

\begin{tabular}{lcc}
\hline$s$ & $\epsilon_{1}(X)$ & $\epsilon_{1}(Y)$ \\
\hline 0 & $9.78137 E-02$ & $1.18408 E-01$ \\
2 & $1.11953 E-01$ & $9.96781 E-02$ \\
4 & $2.16876 E-02$ & $1.76104 E-02$ \\
6 & $3.22178 E-03$ & $2.84069 E-03$ \\
8 & $4.83451 E-04$ & $4.38916 E-04$ \\
10 & $7.50050 E-05$ & $6.69635 E-05$ \\
12 & $1.08812 E-05$ & $1.00510 E-05$ \\
14 & $1.70240 E-06$ & $1.53219 E-06$ \\
16 & $2.59464 E-07$ & $2.32406 E-07$ \\
18 & $3.81681 E-08$ & $3.49836 E-08$ \\
\hline
\end{tabular}

By using Algorithms 3 and 6, we have

$$
\begin{gathered}
X=\left(\begin{array}{cc}
0.497814 & -0.0839355 \\
-0.0839355 & 0.420533
\end{array}\right), \\
Y=\left(\begin{array}{cc}
0.333326 & -0.00556924 \\
-0.00556924 & 0.281592
\end{array}\right), \\
\lambda_{r}(X)=\{0.551576,0.36677\}, \\
\mu_{r}(Y)=\{0.333919,0.280999\} .
\end{gathered}
$$

The results are given in Table 3 . 
TABLE 4: Error analysis for Example 10.

\begin{tabular}{lcc}
\hline$s$ & $\epsilon_{1}(X)$ & $\epsilon_{1}(Y)$ \\
\hline 0 & $6.49247 E-01$ & $2.37069 E-01$ \\
2 & $8.46092 E-02$ & $1.48180 E-01$ \\
4 & $3.51586 E-03$ & $5.04374 E-02$ \\
6 & $8.98887 E-04$ & $1.30368 E-02$ \\
8 & $3.32057 E-04$ & $4.71441 E-03$ \\
10 & $1.12763 E-04$ & $1.57391 E-03$ \\
12 & $3.78219 E-05$ & $5.11273 E-04$ \\
14 & $1.24881 E-05$ & $1.64362 E-04$ \\
16 & $4.06906 E-06$ & $5.26213 E-05$ \\
18 & $1.31421 E-06$ & $1.68193 E-05$ \\
20 & $4.22232 E-07$ & $5.37246 E-06$ \\
22 & $1.35257 E-07$ & $1.71563 E-06$ \\
24 & $4.32590 E-08$ & $5.47811 E-07$ \\
26 & $1.38239 E-08$ & $1.74912 E-07$ \\
28 & $4.41568 E-09$ & $5.58474 E-08$ \\
\hline
\end{tabular}

Example 10. Consider Sys. (1) with $n=2, m=1, \beta=0.65$, and matrices

$$
A=\left(\begin{array}{ccc}
-0.2 & -0.4 & -0.4 \\
-0.6 & -0.2 & 0.6 \\
0.2 & -0.4 & 0.1
\end{array}\right), \quad B=\left(\begin{array}{ccc}
-0.03 & -0.08 & 0 \\
0 & 0 & 0.02 \\
0 & 0.1 & 0
\end{array}\right)
$$

By using Algorithms 3 and 6, we have

$$
\begin{gathered}
X=\left(\begin{array}{ccc}
0.0573078 & -0.000371142 & -0.00702446 \\
-0.000371142 & 0.00075295 & 0.0000206791 \\
-0.00702446 & 0.0000206791 & 0.000896794
\end{array}\right), \\
Y=\left(\begin{array}{ccc}
0.596078 & -0.0233679 & 0.0748523 \\
-0.0233679 & 0.887069 & 0.0104592 \\
0.0748523 & 0.0104592 & 0.454549
\end{array}\right), \\
\lambda_{r}(X)=\{0.0581718,0.000751403,0.0000343988\} \\
\mu_{r}(Y)=\{0.888982,0.627173,0.42154\}
\end{gathered}
$$

The results are given in Table 4 .

\section{Conclusion}

In this paper, the positive definite solutions for Sys. (1) have been tackled. We presented sufficient conditions for the existence of positive definite solutions for Sys. (1). Moreover, we discussed an iterative algorithm from which solutions can always be calculated numerically whenever the system is solvable. Finally, we gave numerical examples that illustrated the behavior of the proposed algorithm.

\section{References}

[1] W. N. Anderson Jr., T. D. Morley, and G. E. Trapp, "Positive solutions to $X=A-B X^{-1} B^{*}$," Linear Algebra and Its Applications, vol. 134, pp. 53-62, 1990.
[2] P. Lancaster and L. Rodman, Algebraic Riccati Equations, Oxford Science, Oxford, UK, 1995.

[3] B. Meini, "Matrix equations and structures: efficient solution of special discrete algebraic Riccati equations," in Proceedings of the WLSSCOO, 2000.

[4] A. M. Aldubiban, Iterative algorithms for computing the positive definite solutions for nonlinear matrix equations [Ph.D. thesis], Riyadh University for Girls, Riyadh, Saudi Arabia, 2008.

[5] A. M. Aldubiban, "Iterative algorithm for solving a system of nonlinear matrix equations," Journal of Applied Mathematics, vol. 2012, Article ID 461407, 15 pages, 2012.

[6] O. L. V. Costa and R. P. Marques, "Maximal and stabilizing Hermitian solutions for discrete-time coupled algebraic Riccati equations," Mathematics of Control, Signals, and Systems, vol. 12, no. 2, pp. 167-195, 1999.

[7] O. L. V. Costa and J. C. C. Aya, "Temporal difference methods for the maximal solution of discrete-time coupled algebraic Riccati equations," Journal of Optimization Theory and Applications, vol. 109, no. 2, pp. 289-309, 2001.

[8] A. Czornik and A. Swierniak, "Lower bounds on the solution of coupled algebraic Riccati equation," Automatica, vol. 37, no. 4, pp. 619-624, 2001.

[9] A. Czornik and A. Świerniak, "Upper bounds on the solution of coupled algebraic Riccati equation," Journal of Inequalities and Applications, vol. 6, no. 4, pp. 373-385, 2001.

[10] R. Davies, P. Shi, and R. Wiltshire, "Upper solution bounds of the continuous and discrete coupled algebraic Riccati equations," Automatica, vol. 44, no. 4, pp. 1088-1096, 2008.

[11] F. Ding and T. Chen, "Iterative least-squares solutions of coupled Sylvester matrix equations," Systems \& Control Letters, vol. 54, no. 2, pp. 95-107, 2005.

[12] F. Ding and T. Chen, "Gradient based iterative algorithms for solving a class of matrix equations," IEEE Transactions on Automatic Control, vol. 50, no. 8, pp. 1216-1221, 2005.

[13] F. Ding and T. Chen, "On iterative solutions of general coupled matrix equations," SIAM Journal on Control and Optimization, vol. 44, no. 6, pp. 2269-2284, 2006.

[14] F. Ding, P. X. Liu, and J. Ding, "Iterative solutions of the generalized Sylvester matrix equations by using the hierarchical identification principle," Applied Mathematics and Computation, vol. 197, no. 1, pp. 41-50, 2008.

[15] B. Hashemi and M. Dehghan, “The interval Lyapunov matrix equation: analytical results and an efficient numerical technique for outer estimation of the united solution set," Mathematical and Computer Modelling, vol. 55, no. 3-4, pp. 622-633, 2012.

[16] J. Ding, Y. Liu, and F. Ding, "Iterative solutions to matrix equations of the form $A_{i} X B_{i}=F_{i}$, Computers \& Mathematics with Applications, vol. 59, no. 11, pp. 3500-3507, 2010.

[17] A. Liu and G. Chen, "On the Hermitian positive definite solutions of nonlinear matrix equation $X^{s}+A^{*} X^{-t_{1}} A+B^{*} X^{-t_{2}} B=$ Q," Mathematical Problems in Engineering, vol. 2011, Article ID 163585, 18 pages, 2011.

[18] L. Xie, J. Ding, and F. Ding, "Gradient based iterative solutions for general linear matrix equations," Computers \& Mathematics with Applications, vol. 58, no. 7, pp. 1441-1448, 2009.

[19] L. Xie, Y. Liu, and H. Yang, "Gradient based and least squares based iterative algorithms for matrix equations $A X B+C X^{T} D=$ F," Applied Mathematics and Computation, vol. 217, no. 5, pp. 2191-2199, 2010.

[20] F. Yin and G.-X. Huang, "An iterative algorithm for the least squares generalized reflexive solutions of the matrix equations 
$A X B=E, C X D=F, "$ Abstract and Applied Analysis, vol. 2012, Article ID 857284, 18 pages, 2012.

[21] W. Zhao, H. Li, X. Liu, and F. Xu, "Necessary and sufficient conditions for the existence of a Hermitian positive definite solution of a type of nonlinear matrix equations," Mathematical Problems in Engineering, vol. 2009, Article ID 672695, 13 pages, 2009.

[22] H. Mukaidani, "Newton's method for solving cross-coupled sign-indefinite algebraic Riccati equations for weakly coupled large-scale systems," Applied Mathematics and Computation, vol. 188, no. 1, pp. 103-115, 2007.

[23] H. Mukaidani, S. Yamamoto, and T. Yamamoto, "A Numerical algorithm for finding solution of cross-coupled algebraic Riccati equations," IEICE Transactions, vol. 91, pp. 682-685, 2008.

[24] H. Mukaidani, "Numerical computation of cross-coupled algebraic Riccati equations related to $H_{\infty}$-constrained LQG control problem," Applied Mathematics and Computation, vol. 199, no. 2, pp. 663-676, 2008.

[25] I. G. Ivanov, "A method to solve the discrete-time coupled algebraic Riccati equations," Applied Mathematics and Computation, vol. 206, no. 1, pp. 34-41, 2008.

[26] I. G. Ivanov, "Stein iterations for the coupled discrete-time Riccati equations," Nonlinear Analysis. Theory, Methods \& Applications, vol. 71, no. 12, pp. 6244-6253, 2009.

[27] R. Bhatia, Matrix Analysis, vol. 169 of Graduate Texts in Mathematics, Springer, New York, NY, USA, 1997.

[28] M. Fujii and Y. Seo, "Reverse inequalities of Araki, Cordes and Löwner-Heinz inequalities," Nihonkai Mathematical Journal, vol. 16, no. 2, pp. 145-154, 2005.

[29] P. Lancaster, Theory of Matrices, Academic Press, New York, NY, USA, 1969.

[30] C.-H. Guo and N. J. Higham, "A Schur-Newton method for the matrix $p$ th root and its inverse," SIAM Journal on Matrix Analysis and Applications, vol. 28, no. 3, pp. 788-804, 2006. 


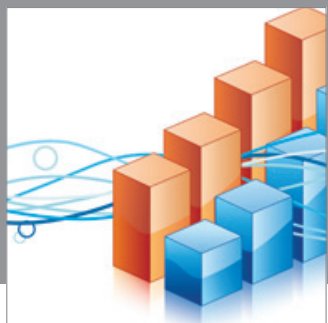

Advances in

Operations Research

mansans

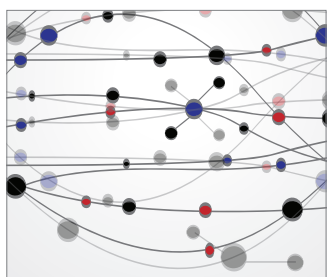

The Scientific World Journal
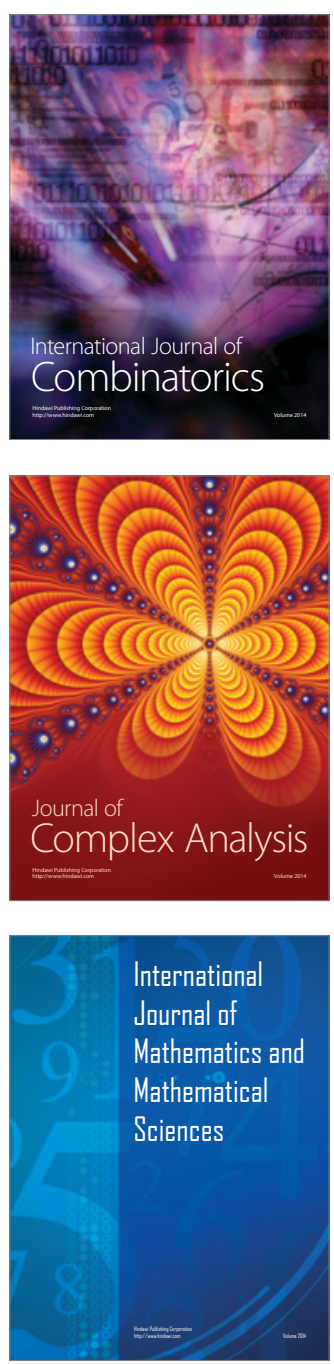
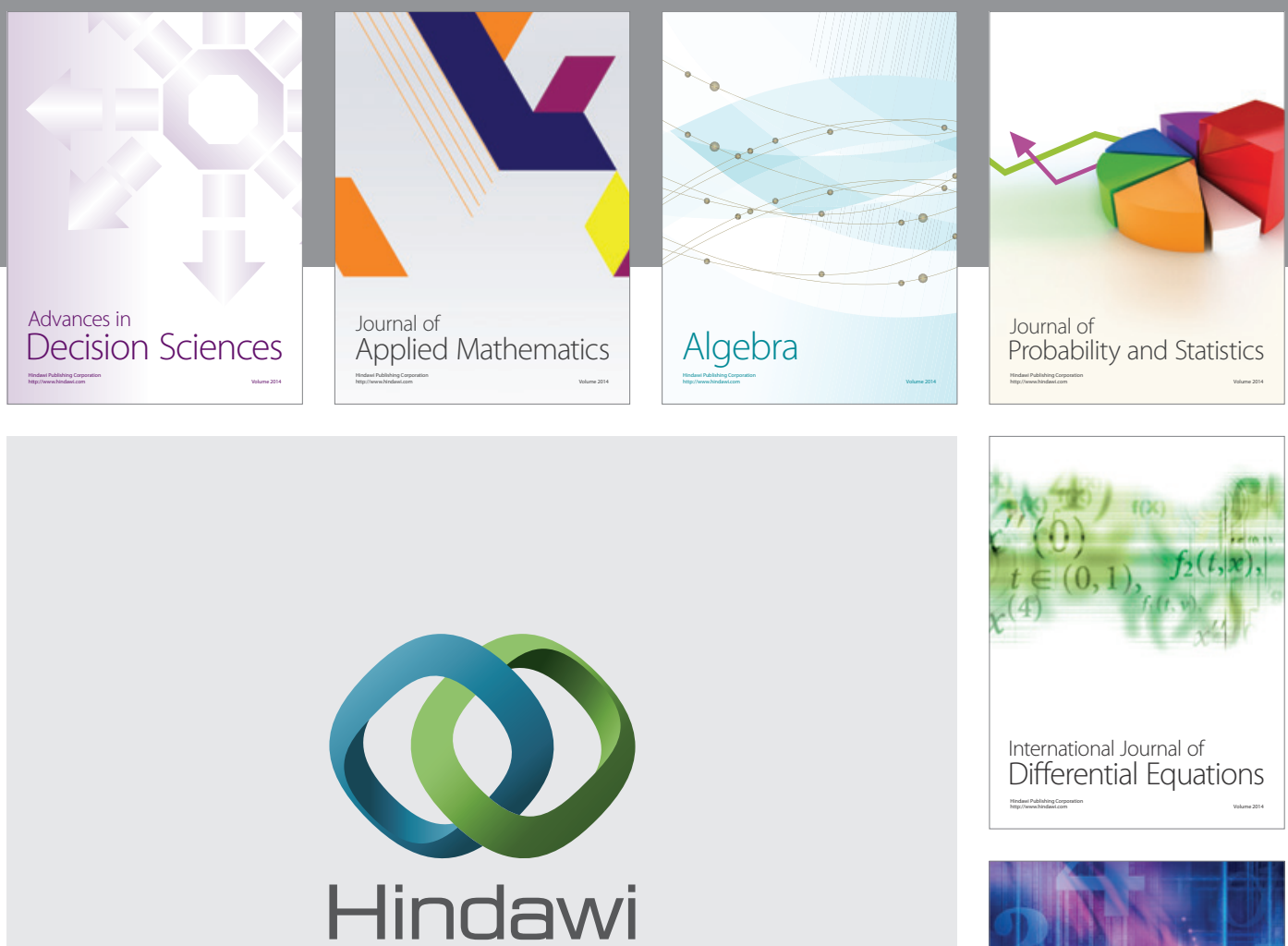

Submit your manuscripts at http://www.hindawi.com
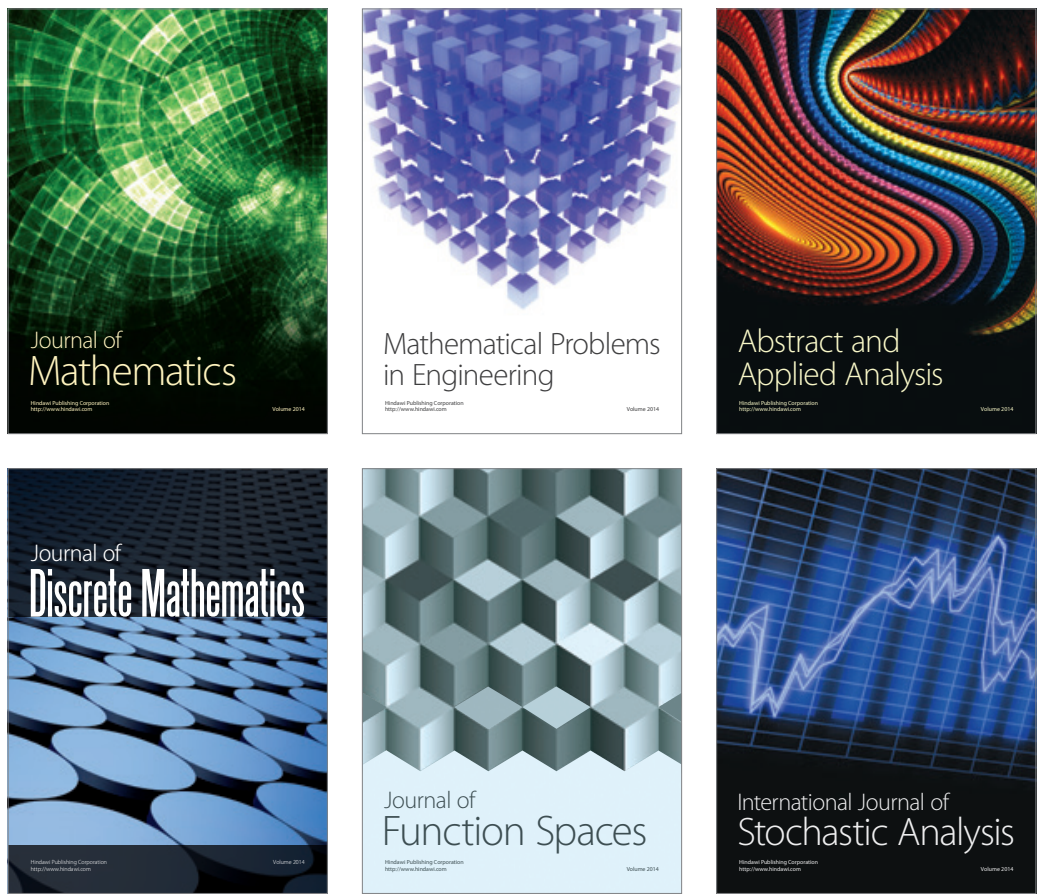

Journal of

Function Spaces

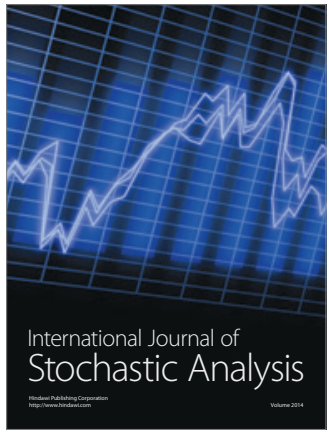

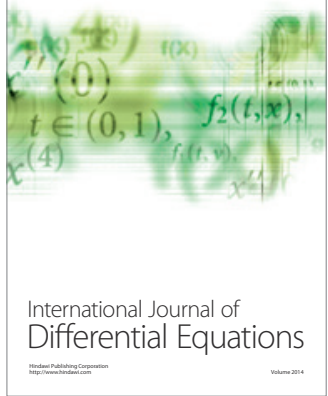
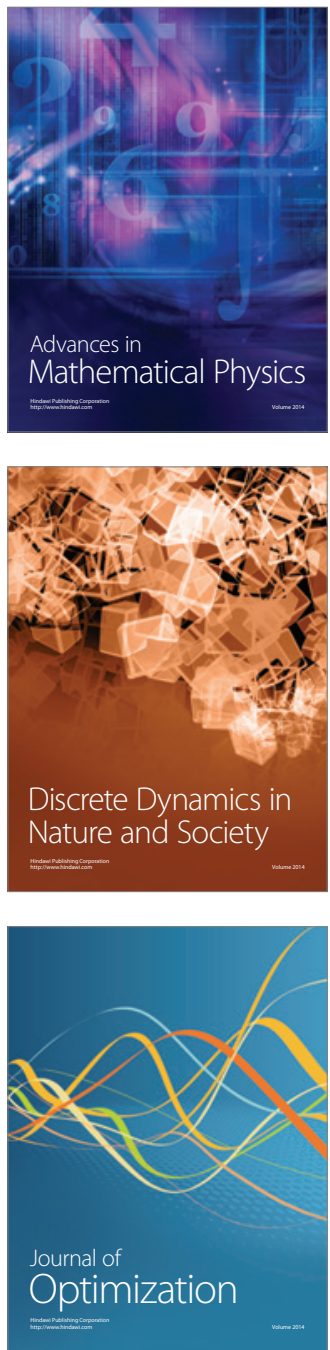